\title{
La recombinaison homologue : mécanismes et conséquences
}

\author{
Jean-Luc Rossignol
}

Professeur à l'université Paris-Sud, Institut de génétique et microbiologie, bt 400, Cnrs URA 1354, université Paris-Sud, 91405 Orsay Cedex, France.

\section{Les processus de recombinaison}

La recombinaison génétique désigne les processus qui conduisent à changer l'association physique entre deux segments d'ADN. Elle joue un rôle fondamental à la fois dans la diversification des génomes et dans le maintien de leur homogénéité. Elle est de plus en plus utilisée comme outil pour effectuer des modifications contrôlées des génomes et pour construire de nouvelles macromolécules biologiques. Les approches moléculaires les plus récentes soulignent la grande diversité des mécanismes de recombinaison. Ceux-ci peuvent être classés en quatre grands groupes: recombinaison homologue, transposition, recombinaison site-spécifique et recombinaison illégitime. La recombinaison homologue (aussi appelée recombinaison générale) ṇécessite une homologie de séquence relativement importante entre les deux partenaires. Lorsque l'étendue de cette homologie descend au-dessous de quelques dizaines de paires de bases chez les procaryotes et de 200 paires de bases chez les mammifères (dans le cas de recombinaisons intra- ou interplasmidiques), la fréquence de recombinaison diminue de manière brutale [1]. Une autre caractéristique de la recombinaison homologue est qu'elle peut se produire n'importe où dans le génome. Pourtant, elle n'est pas forcément équiprobable, certaines régions du génome pouvant être plus fréquemment impliquées que d'autres dans des événements de recombinaison. La transposition implique des séquences particulières capables de se déplacer d'un point à l'autre du génome et qui portent l'information nécessaire à leur mouvement. La recombinaison site-spécifique correspond à des événements de recombinaison program- més s'effectuant en des positions très précises des génomes. La recombinaison illégitime regroupe les types de recombinaison ne répondant pas aux définitions précédentes. On se bornera ici à envisager quelques aspects de la recombinaison homologue, qui se produit chez tous les organismes vivants.

La recombinaison homologue reflète une variété de situations

La recombinaison homologue peut s'effectuer dans des situations variées qui peuvent être distinguées sur deux critères : la nature des partenaires et le moment du cycle cellulaire pendant lequel elle se produit.

\section{Variété des partenaires}

Les deux segments d'ADN qui vont interagir dans l'acte de recombinaison peuvent être situés sur la même molécule (recombinaison intramoléculaire) ou bien sur des molécules distinctes (recombinaison intermoléculaire). Ces molécules peuvent être par exemple deux chromosomes, deux plasmides ou bien un plasmide et un chromosome, deux virus, un phage et un prophage, etc.

La recombinaison homologue entre plasmide et chromosome (et plus précisément entre l'ADN exogène introduit dans la cellule par transfection et le chromosome de l'hôte) constitue un problème très actuel. L'enjeu de l'introduction d'ADN exogène est de remplacer de manière contrôlée un gène cellulaire par le même gène dans lequel une mutation a été préalablement introduite par mutagenèse in vitro. Pour cela, le gène apporté par
l'ADN transfectant doit s'intégrer à la place du gène résident. Chez les eucaryotes supérieurs, l'ADN transfectant s'intègre le plus souvent de manière non homologue, en des endroits variés du génome de la cellule hôte. Diverses méthodes permettent d'augmenter l'efficacité des recombinaisons homologues et de sélectionner les cellules dans lesquelles a eu lieu le remplacement de gène [2]. $\mathrm{La}$ recombinaison entre chromosomes peut correspondre à divers types d'interactions. Les séquences qui interagissent peuvent être situées au même locus (recombinaison homotopique), dans ce cas, les deux séquences peuvent être situées sur des chromosomes homologues et sont alors susceptibles de présenter des différences, ou bien être situées sur deux chromatides sœurs, elles sont alors tout à fait identiques, issues par réplication d'un même duplex parental. Les séquences qui interagissent peuvent aussi être situées à des emplacements différents (recombinaison ectopique), si la cellule comporte des séquences répétées. Si les séquences répétées sont situées sur le même chromosome, la recombinaison peut être intramoléculaire ou intermoléculaire (entre chromatides sœurs ou entre chromosomes homologues).

Recombinaison méiotique et recombinaison mitotique

Le moment privilégié du cycle cellulaire où s'effectue la recombinaison homologue est la prophase de méiose, après la réplication préméiotique (recombinaison méiotique). Néanmoins, la recombinaison peut s'effectuer dans les cellules effectuant des mitoses. Elle peut alors avoir lieu avant ou après la synthèse d'ADN (phase G1 ou G2). 


\section{Conversion génique et crossing-over}

La recombinaison homologue aboutit ì deux types de produits, qui ne peuvent être distingués l'un de l'autre que si l'on est en mesure de déterminer simultanćment l'information génétique portée par les deux partenaires avant et après l'actc de recombinaison :

- Les conversions géniques, qui correspondent à un transfert unidirectionnel d'information d'un duplex parental vers l'autre (figure $1 A$ ). Ce sont des événements localisés dont la taille varie entre quelques paires et quelques milliers de paires de bases ;

- Les crossing-over, qui correspondent à un échange réciproque des segments d'ADN situés de part et d'autre de la région d'interaction (figure $1 B$ ). On distinguera le crossing-over, qui désigne un événement moléculaire, du chiasma, qui désigne une figure cytologique, c'est-àdire un changement réciproque de partenaires entre chromatides dans les paires de chromosomes homologues appariés en fin de prophase de méiose. Les chiasmas résultent des crossing-over, ils en sont les reflets cytologiques.

\section{Les intermédiaires de recombinaison}

Une caractéristique essentielle du processus de recombinaison homologue consiste dans la formation d'intermédiaires de recombinaison correspondant à des molécules parentales jointes par l'intermédiaire de liaisons hydrogène entre deux brins complémentaires provenant chacun d'une molécule parentale différente (ADN hybride). Les modèles moléculaires de recombinaison sont variés, car ils supposent divers événements initiateurs (cassure double-brin (figure $2 A$ ), coupure simplebrin (figure $2 B$ ), ou extension d'une brèche sur un seul brin (figure 2C), par exemple). Tous ces événements sont susceptibles de conduire à des molécules jointes par l'intermédiaire de régions d'ADN hybride. La maturation de ces molécules jointes aboutirait aux conversions et aux crossing-over.

\section{Deux mécanismes de conversion}

La formation d'une coupure double-brin sur une des molécules partenaires, suivie de l'extension d'une brèche double-brin (figure $2 A$ ) constitue un premier mécanisme de conversion. La brèche est comblée par une réplication réparative qui prend pour modèle le duplex intact (figure 3). La molécule d'ADN initialement coupée acquiert à l'issue de sa réparation l'information génétique de l'autre parent : elle est convertie. L'existence de ce type de processus a été démontrée par des expériences de transformation de la $\mathrm{m} / \mathrm{s} n^{\circ} 7$ vol. 6 , septembre 90

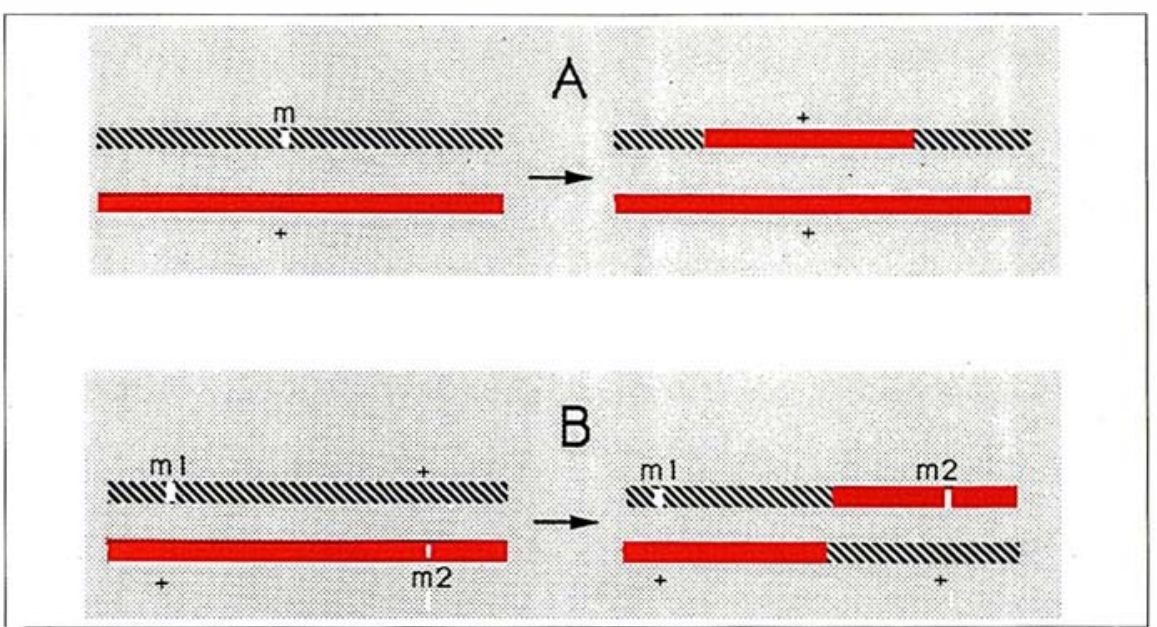

Figure 1. Les produits de la recombinaison homologue. Chaque trait symbolise un ADN duplex. A - La conversion génique résulte d'un transfert unidirectionnel d'information depuis une molécule vers l'autre. $B$ - Le crossing-over résulte d'un échange réciproque d'information entre les deux molécules parentales.

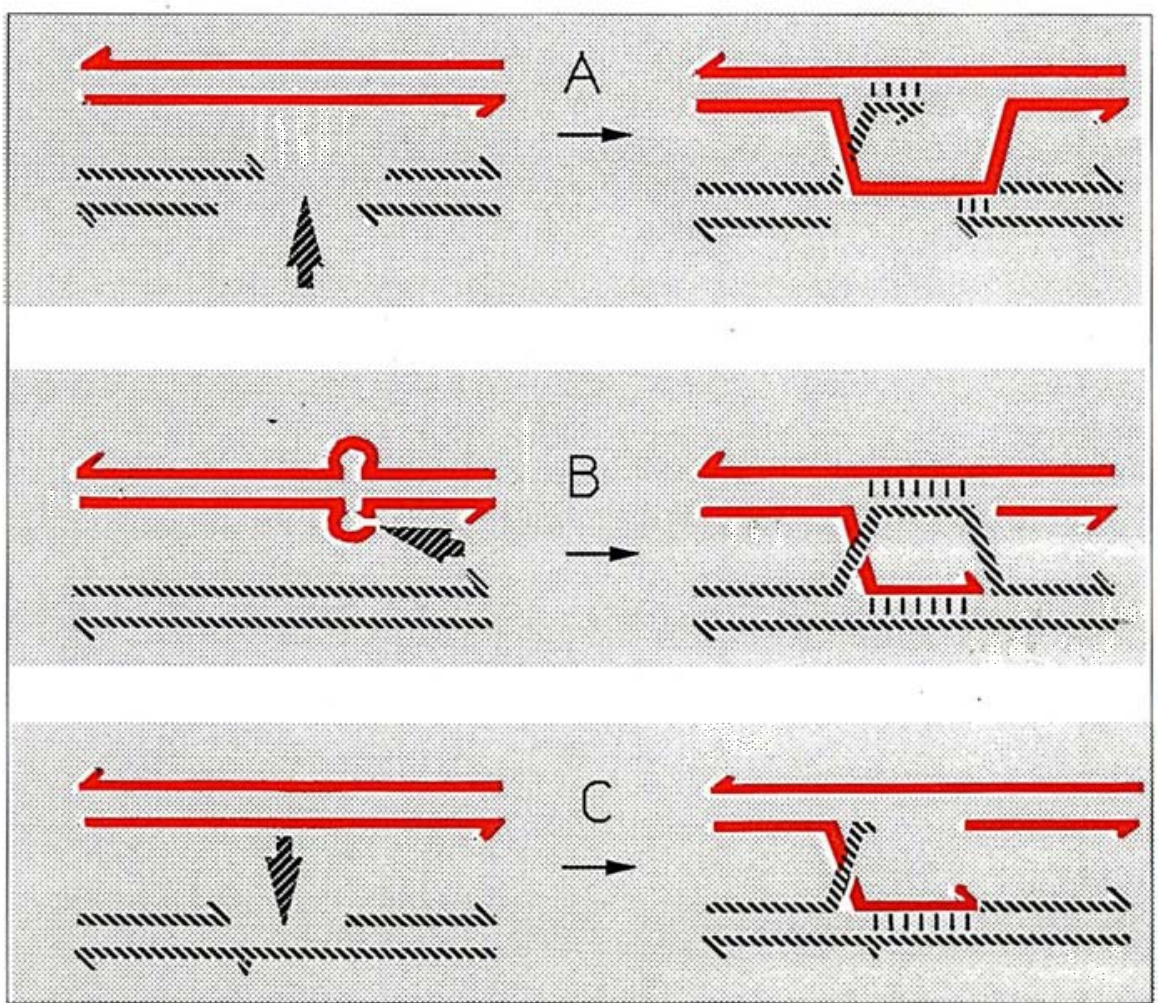

Figure 2. Intermédiaires de recombinaison dans trois modèles de recombinaison homologue. Ces modèles se distinguent par la nature des intermédiaires initiant la recombinaison (à gauche) et par la nature des intermédiaires correspondant à des molécules jointes (à droite). Ils présentent deux points communs: 1) les molécules jointes sont associées par des segments d'ADN hybride réunis sant par l'intermédiaire de liaisons hydrogène deux brins complémentaires, issus chacun d'une molécule parentale ; 2) à la jonction entre les deux molécules se forme une connexion croisée de brins (en forme de $X$ sur les schémas) dont on pense que la résolution peut conduire auX crossing-over. A - Réparation de cassures double-brin (d'après Szostak et al., 1983, Cell, 33: 25); B - Recombinaison par la voie binaison par la vole reCBCD chez E. Coli, initiee par une cassure simple-brin (d'apres Smith et al. Cold Spring Harbor Symp. Quant. Biol., vol. 49: 487). C - Reparation de breches postréplicatives simple-brin provoquées par un dimère de pyrimidine ld'après Howard-Flanders et al., 1984, Nature, $309: 2151$ 
levure Saccharomyces cerevisiae avec des plasmides comportant une brèche double-brin effectuée in vitro: cette brèche est réparée par copie de la région homologue située dans le chromosome de l'hôte [3]. Un second mécanisme de conversion résulte de la formation de régions d'ADN hybride dans les molécules jointes. Si les deux partenaires diffèrent par une ou plusieurs mutations dans la région d'ADN hybride, celle-ci comportera a u niveau de ces mutations des mésappariements consécutifs à la non-complémentarité des bases (ADN hétéroduplex). L'existence de tels segments hétéroduplex a été démontrée génétiquement [4] et physiquement [5]. Ces hétéroduplex constituent une seconde source de conversion. Il y a plusieurs possibilités selon que les mésappariements persistent jusqu'à la réplication ou sont préalablement corrigés (figure 4). S'ils persistent, la conversion sera installée sur la longueur du brin transféré après un cycle de réplication. S'il y a correction par excision d'un long segment d'ADN simple-brin, suivi d'une réparation qui comble la brèche ainsi créée, on obtiendra d'emblée la conversion, sans réplication, si le brin excisé est le brin résident. S'il y a plusieurs corrections indépendantes par excision de courts segments sur l'un ou l'autre brin, on obtiendra des conversions multiples associées.

Il est important de noter que l'absence de correction des ADN hétéroduplex et les corrections de grande taille homogénéisent l'information. Au contraire, les corrections courtes engendrent de la diversité.

L'existence de corrections par excision de longs et de courts segments a été démontrée chez les bactéries par différents groupes [6]. L'existence de corrections est aussi démontrée chez les eucaryotes, mais leur étude est moins avancée.

\section{L'origine des crossing-over}

La réponse à la question de l'origine des crossing-over doit tenir compte de la forte association qui est généralement observée au cours de la recombinaison homologue entre conversion et crossing-over : quand on sélectionne, chez les champignons, les conversions pour un marqueur génétique, on observe qu'elles sont souvent associées avec un crossing-over. Pour rendre compte de cette observation, tous les modèles moléculaires de recombinaison supposent que crossing-over et conversion résultent de la même suite d'événements moléculaires. $\mathrm{La}$ maturation des intermédiaires de recombinaison qui conduisent aux conversions (figure 2) nécessite des coupures endonucléotidiques qui permettent la ces coupures préservent les associations parentales de part et d'autre de la région de conversion, ou bien conduisent à un crossing-over.

Rien ne permet pourtant d'exclure l'hypothèse selon laquelle crossing-over et conversion résulteraient d'événements moléculaires distincts. Le premier événement (par exemple la conversion) pourrait laisser une trace qui favoriserait l'occurrence d'un second événement (par exemple le crossing-over) qui s'effectuerait alors à la même place.

Rôles différents des recombinaisons méiotique et mitotique

Chez les eucaryotes, les recombinaisons homologues s'effectuent essentiellement pendant la prophase de méiose ; on met en évidence également des recombinaisons mitotiques, plus rares. Les propriétés comparées des recombinaisons méiotique et mitotique [7] suggèrent qu'elles jouent des rôles différents, qui ne se limitent pas au brassage des gènes.

1) $\mathrm{La}$ fréquence des recombinaisons méiotiques est élevée. Ainsi, il y a au moins un crossing-over, et quelquefois plusieurs, par bras chromosomique et par cellule. Les recombinaisons mitotiques ont été bien étudiées chez la levure $S$. cerevisiae. Elles sont environ mille fois plus rares.

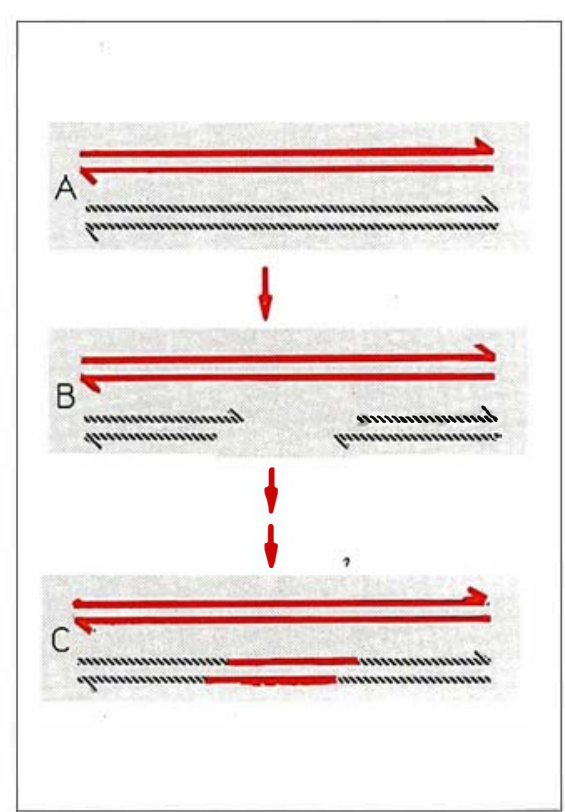

Figure 3. Conversion par réparation de cassures double-brin. $A-$ Duplex parentaux. $B$ - Formation d'une brèche double-brin sur un duplex. C - Après plusieurs étapes (passant par
la formation de molécules jointes: voir figure 2A), la brèche est comblée par copie de l'information du duplex intact.
2) Les recombinaisons méiotiques ne sont pas réparties également le long du génome : la probabilité de recombinaison varie d'un facteur proche de 100 d'une région à l'autre du génome chez les champignons. Cela est attesté en particulier par la très forte variation des fréquences moyennes de conversions d'un gène à l'autre [8]. La distribution dans le génome des recombinaisons mitotiques est différente [7]. Il serait intéressant de rechercher si les recombinaisons mitotiques s'effectuent ou non de manière aléatoire.

3) Les recombinaisons méiotiques sont initiées en des sites spécifiques précis. Cela se fonde sur trois types d'observations :

- Les études génétiques de la conversion montrent qu'il s'agit d'un événement polarisé qui s'étend à partir d'une extrémité du gène [9]. Cela suggère que l'événement de recombinaison présente une extrémité fixe qui correspond à un site d'initiation spécifique ;

- Une étude par délétion a permis de déterminer une région de 279 paires de bases qui contient un élément dont la présence est nécessaire aux conversions dans le gène $A R G 4$ de levure [10]. Cet élément, situé à l'extrémité du gène qui présente une haute fréquence de conversions, a les propriétés d'un site d'initiation préférentiel.

- Une étude moléculaire de l'ADN dans les méioses synchrones de levure a montré, au niveau du gène $A R G 4$, l'apparition de cassures double-brin qui sont localisées au niveau de l'élément d'initiation de conversion [11].

En revanche, lors de la recombinaison mitotique, rien n'indique l'existence de sites privilégiés d'initiation de la recombinaison. En particulier, il n'a pas été observé de polarité de conversion [7].

Les caractéristiques de la recombinaison méiotique indiquent que sa fréquence, sa localisation et son initiation sont soumises à un contrôle précis. Par opposition, la recombinaison mitotique n'est pas initiée en des sites spécifiques. Ces différencs reflètent probablement les rôles très différents de ces deux types de recombinaison.

Recombinaison mitotique et réparation des lésions de l'ADN

La fréquence des recombinaisons mitotiques est fortement augmentée par les traitements qui endommagent l'ADN (rayons ionisants, rayons UV). La capacité à effectuer des recombinaisons mitotiques semble être un processus inductible [12]. Ces observations suggèrent qu'il existe une condition limitante pour la recombinaison mitotique qui ne serait pas rem- 


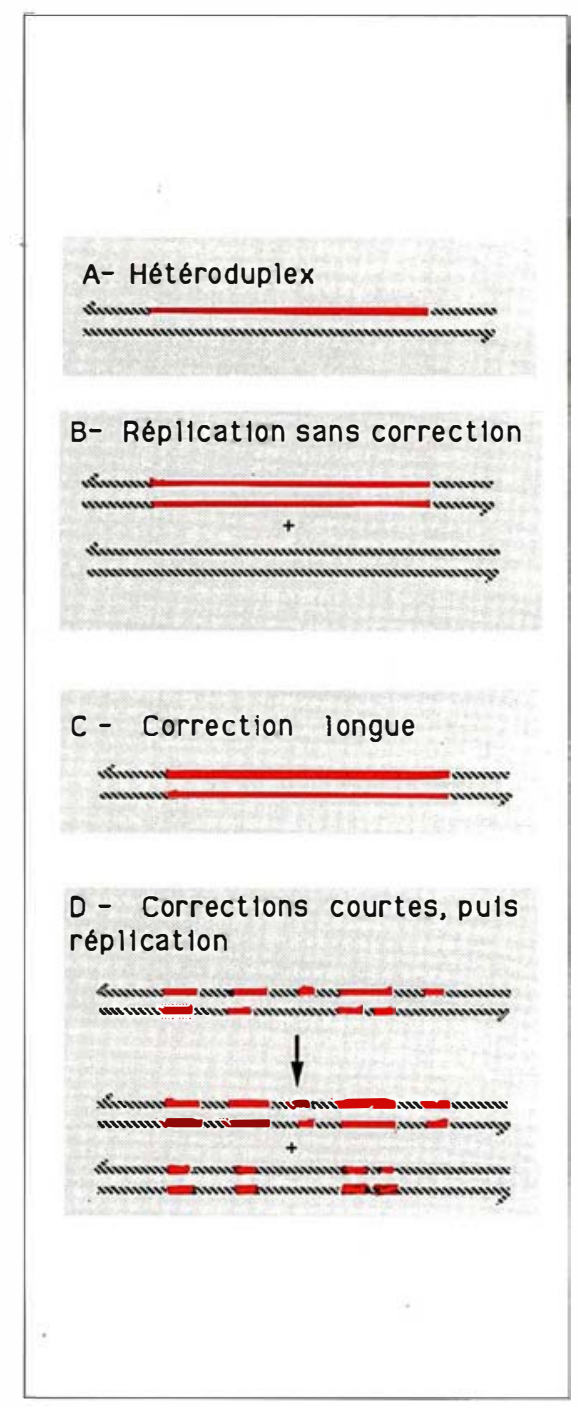

Figure 4. Conversion par formation d'hétéroduplex et correction de mésappariements. A - Hétéroduplex (brins résidents en hachuré brin transféré en rougel. B L'hétéroduplex est brin transfére en rouge. B-L heteroduplex est répliqué sans avoir été corrigé, le transfert d'information est effectif sur les deux brins d'une des molécules filles. C - L'hétéroduplex est corrigé par excision d'un long segment du brin résident, suivie par une resynthèse qui copie l'information portée par le brin transféré : on obtient d'emblée un transfert d'information sur les deux brins de la molécule sans réplication préalable. D - Plusieurs mésappariements sont corrigés de - Plusieurs mésappariements sont corrigés de maniere independante par excision-resynthèse de courts segments du prin résident ou du brin transféré. Après réplication, on obtient plusieurs transferts d'information associés, impliquant chacun un court segment $d^{\prime} A D N$. plie dans la majorité des cellules. Cette condition pourrait être induite dans une fraction des cellules par des lésions dans l'ADN et contribuerait à réparer ces lésions.

Recombinaison méiotique, appariement et disjonction des chromosomes

Dans les cellules en méiose, les crossingover semblent jouer un rôle dans la bonne disjonction des chromosomes [13]. Une question centrale est celle des relations entre l'appariement méiotique des chromosomes homologues et la recombinaison. La vision classique suppose qu'au cours de la prophase de méiose, les chromosomes homologues s'alignent, amenant les séquences d'ADN homologues en contact, ce qui permettrait leur recombinaison. Cette vision est probablement simpliste : l'observation au microscope électronique des structures d'appariement indique que les $\mathrm{ADN}$ des chromosomes homologues sont séparés d'environ $100 \mathrm{~nm}$ : seule une toute petite fraction de l'ADN doit présenter un appariement effectif.

L'étude des effets sur l'appariement chromosomique et la recombinaison de mutants du gène $R A D 50$ de $S$. cerevisiae, dont le produit est requis pour les deux processus, suggère une interprétation alternative [14]. La première étape consisterait en une recherche d'homologie qui pourrait requérir l'existence préalable des événements initiateurs de la recombinaison méiotique (probablement des coupures double-brin spécifiques, comme on l'a vu plus haut). Cela aboutirait à des appariements ponctuels entre les homologues par l'intermédiaire de liaisons hydrogène entre les brins complémentaires appartenant à des molécules homologues. C'est au niveau de ces appariements ADN/ADN très localisés, qui assurent la reconnaissance entre les homologues, que les recombinaisons s'effectueraient. Ainsi, la structure d'appariement au niveau chromosomique pourrait se mettre en place de proche en proche. Dans cette interprétation, le processus d'initiation de la recombinaison serait simultanément responsable de l'appariement méiotique des chromosomes homologues et de l'échange d'information génétique.

Conversion ectopique, homogénéisation et diversification des séquences répétées

Les recombinaisons ectopiques (entre séquences répétées) ont été observées dans de très nombreux systèmes expérimentaux. Chez les eucaryotes supérieurs, elles sont aussi inférées à partir de comparaisons de séquences.
Les conversions et les crossing-over ectopiques ont des conséquences très différentes. Les conversions peuvent avoir pour effet d'homogénéiser les séquences répétées. Ainsi, elles sont invoquées comme mécanisme possible du molecular drive, c'est-à-dire de l'observation que les séquences répétées à l'intérieur d'une même espèce divergent moins entre elles qu'avec les séquences répétées de la même famille d'une espèce voisine. Les conversions peuvent aussi engendrer la diversité (figure 4). C'est le cas dans certains types de recombinaison spécialisée, comme celle qui produit la région variable des immunoglobulines de poulet [15]. Un rôle des conversions dans la genèse des régions hypervariables des chaînes d'immunoglobulines de souris n'est pas exclu [16].

\section{Crossing-over ectopiques et réarrange- ments chromosomiques}

Les crossing-over ectopiques entraînent des réarrangements chromosomiques. Pour les séquences répétées en tandem, ils conduisent à une variation du nombre de copies. Dans le cas des ADN ribosomiques de $S$. cerevisiae, de fréquents crossingover inégaux (figure $5 \mathrm{~A}$ ) entre chromatides sœurs (environ $1 \%$ par cellule et par génération) entraînent une variation du nombre de copies [17]. Il n'est pas démontré que les crossing-over inégaux opèrent avec des fréquences significatives dans les autres cas de séquences répétées en tandem. Les crossing-over entre séquences répétées dispersées engendrent quant à eux des délétions ou des inversions quand ces séquences sont situées sur le même chromosome (figures $5 B, 5 C$ ) et des translocations réciproques ou des chromosomes dicentriques et acentriques quand elles sont situées sur des chromosomes différents (figures $5 D, 5 E$ ).

Pourquoi les recombinaisons ectopiques ne conduisent-elles pas à l'éclatement des génomes?

S'ils étaient fréquents, ces crossing-over ectopiques conduiraient à un éclatement du génome, consécutif à de multiples réarrangements chromosomiques, surtout chez les eucaryotes supérieurs où ces génomes comportent des séquences répétées un très grand nombre de fois. La question fondamentale est de comprendre pourquoi les recombinaisons entre séquences répétées sont si rares. De fait, les recombinaisons mitotiques sont naturellement rares, ce qui peut suffire à maintenir la fréquence des recombinaisons ectopiques à des taux tolérables par l'organisme. Mais qu'en est-il en méiose 
où les recombinaisons sont beaucoup plus fréquentes? Plusieurs hypothèses peuvent être avancées :

1) L'alignement des chromosomes homologues est préalable à la recombinaison et interdit donc la reconnaissance entre séquences ectopiques. Cette interprétation est réfutée par des expériences effectuées chez la levure [18]. La recombinaison méiotique entre séquences répétées artificielles est aussi fréquente qu'entre séquences situées au même emplacement, et présente une forte proportion de crossing-over (qui aboutissent à des réarrangements chromosomiques). Cette observation indique clairement qu'en ellemême, la position relative des séquences homologues (en un même site ou en des sites différents) n'a pas d'influence sur leur recombinaison. Si les séquences répétées qui existent naturellement dans le génome recombinaient aussi fréquemment entre elles que les séquences répétées artificielles étudiées dans ces expériences, pratiquement tous les produits de méiose subiraient un réarrangement. Cela n'est pas observé. Un autre mécanisme doit donc empêcher la recombinaison méiotique entre séquences répétées.

2) La divergence naturelle entre les séquences répétées constitue une barrière à la recombinaison. Dans cette hypothèse, proposée par M. Radman [19], lors de la recombinaison entre séquences divergentes, les mésappariements formés sur les régions hétéroduplex qui joignient les deux molécules sont reconnus par les systèmes de correction. Ceux-ci empêcheraient la stabilisation des hétéroduplex et feraient avorter les recombinaisons. Cette hypothèse est supportée par des expériences qui montrent que des mutations qui inactivent le système de correction permettent de rétablir la recombinaison entre des espèces voisines de bactéries qui présentent un taux de divergence d'environ $20 \%$ dans leurs séquences d'ADN [19]. Cette hypothèse a encore besoin d'être confirmée expérimentalement en ce qui concerne les séquences répétées chez les eucaryotes.

3) Les séquences répétées naturelles sont situées dans des régions froides pour la recombinaison méiotique. Dans cette hypothèse, les séquences répétées dans les régions où la recombinaison est fréquente seraient éliminées du fait de leur désavantage sélectif.

4) Les séquences répétées naturelles ne comportent pas d'élément d'initiation de recombinaison méiotique. Cette hypothèse, comme la précédente, prédit que les recombinaisons homotopiques méiotiques entre séquences répétées sont rares. 5) Des modifications épigénétiques préméiotiques affectant spécifiquement les séquences répétées empêchent celles-ci de recombiner. On sait par exemple que la

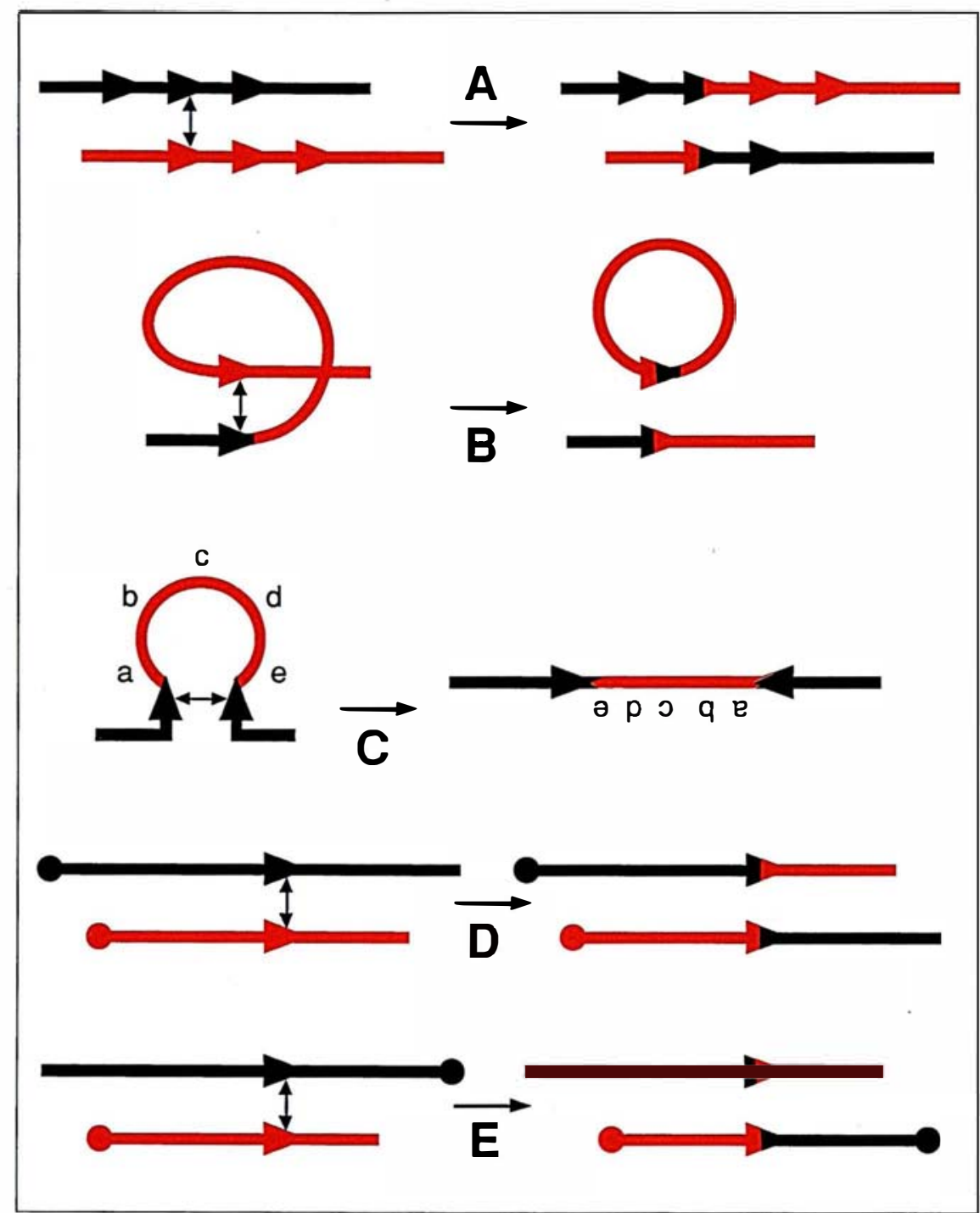

Figure 5. Réarrangements chromosomiques consécutifs aux crossing-over ectopiques. Les flèches représentent les séquences répétées et le sens des répétitions. A - Variation du nombre de copies des régions répétées par crossing-over inégaux entre chromatides sœurs. $B$ - Délétion d'un segment d'ADN par crossing-over entre séquences répétées flanquantes de même orientation. $C$ - Inversion d'un segment $d^{\prime} A D N$ par crossing-over entre séquences répétées flanquantes en orientation inversée. D - Translocation réciproque par crossing-over entre séquences répétées situées cur dition sur deux chromos.omes différents et orientés dans respectif. E - Genèse de chromosomes acentriques et dicentriques par crossing-over entre séquences répétées situées sur deux chromosomes différents, en orientation inversée par rapport à leur centromere respectif.

méthylation de l'ADN en des sites spécifiques peut interférer avec sa coupure par des enzymes de restriction, ou bien avec sa transcription : si des modifications de l'ADN affectent sa reconnaissance par des protéines qui se lient avec lui, elles pourraient aussi empêcher sa recombinaison. La possibilité d'un tel processus est suggérée de manière indirecte par les phénomènes de méthylation de séquences répétées artificielles observées chez deux champignons filamenteux, Neurospora crassa [20] et Ascobolus immersus [21]. Des séquences artificiellement dupliquées sont sujettes avant la méiose à une méthyla- tion de leurs cytosines. Chez Neurospora crassa, la méthylation des cytosines est associée de surplus à une hypermutabilité qui correspond à une transition de $\mathrm{C}$ $\mathrm{G}$ vers $\mathrm{T}$-A de $10 \%$ à plus de $20 \%$ des C-G [20]. A la différence de Neurospora, il n'y a pas de mutagenèse chez Ascobolus. Une caractéristique intéressante de ce processus est qu'il nécessite probablement une étape de reconnaissance entre les séquences répétées, qui doit impliquer leur appariement [22]. Quelle est la signification biologique et quelles sont les conséquences de ce processus de méthylation? Une des hypothèses est qu'il pour- 
rait être un moyen pour la cellule d'empêcher les recombinaisons méiotiques entre les séquences répétées naturelles. Ces différentes hypothèses, qui rendraient compte d'un faible niveau de recombinaison des séquences répétées, ne s'excluent pas mutuellement. D'un organisme à l'autre, les hasards évolutifs peuvent avoir mis à la disposition des cellules des moyens différents de prévenir ces recombinaisons. Tout moyen efficace, s'i n'entraîne pas par ailleurs d'inconvénient majeur, devrait conférer aux cellules un sérieux avantage sélectif. Ainsi, il est peu probable que les levures - qui ne possèdent apparemment pas de système de méthylation - utilisent une stratégie de méthylation préméiotique des séquences répétées, mais elles pourraient avoir développé un ou plusieurs autres systèmes. Il peut être plus économique pour un système biologique de développer simultanément plusieurs mécanismes de conservation de l'information génétique (dans notre cas, le maintien de l'intégrité du génome) que d'en élaborer un seul ayant une très grande efficacité. Cela est bien montré, par exemple, avec les processus de fidélité de la réplication [23]. Il ne serait pas étonnant que dans un même organisme se soient développés simultanément plusieurs systèmes de contrôle de la recombinaison
1. Subramani S, Seaton BL. Homologous recombination in mitotic dividing mammalian cells. In Smith GR, Kucherlapati R, ed. Genetic Recombination. Washington DC : American Society for Microbiology, 1988 : 549-73.

2. Capecchi $M$. The new mouse genetics : altering the genome by gene targeting. Trends in Genetics $1989 ; 5: 70-6$

3. Orr-Weaver T, Szostak J, Rothstein R. Yeast transformation : a model system for the study of recombination. Proc Natl Acad Sci USA 1981; 78 6354-8.

4. Leblon G, Rossignol J-L. Mechanism of gene conversion in Ascobolus immersus. III. The interaction of heteroalleles in the conversion process. $M o l$ Gen Genetics 1973 ; 122 : 165-82.

5. Lichten M, Goyon C, Schultes NP, et al. Detection of heteroduplex DNA molecules among the products of Saccharomyces cerevisiae meiosis. Proc Natl Acad Sci USA 1990 (sous presse).

6. Radman M. Mismatch repair and genetic recombination. In : Smith GR, Kucherlapati R, ed. Genetic Recombination. Washington DC : American Society for Microbiology, 1988: 169-92. 7. Kunz BA, Haynes RH. Phenomenology and genetic control of mitotic recombination in yeast. In : Wilson JH, ed. Genetic Recombination. The Benjamin/Cummings Publishing Co, Inc, 1985 : 195-227.

8. Nicolas A. Variation of gene conversion and

\section{Summary}

Homologous recombinaison : mecanisms and consequences

Homologous genetic recombination corresponds to a variety of situations and may be accomplished by different pathways. It leads to gene conversion, an unidirectional transfer of information, and crossing-over, a reciprocal exchange of information. Gene conversion may result either from the filling of double strand gaps or from the formation of heteroduplex DNA followed 'by mismatch correction. Crossing-over is frequently associated to the conversion event. This association is currently seen as reflecting the formation of a recombination intermediate which is common to both processes. In eukaryotes, meiotic recombination, the frequency, the repartition and the initiation of which are submitted to a precise control, plays a role in chromosome disjunction and probably in chromosome pairing. Mitotic recombination shows different properties and may be involved in recombination repair of DNA lesions. Recombination can involve homologous sequences which are dispersed within the genome. This generates chromosome rearrangements which are harmful to the cell. Possible processes preventing this recombination between repeated sequences are proposed and discussed.

intragenic recombination frequencies in the genome of Ascobolus immersus. Mol Gen Genetics $1979 ; 176: 129-38$.

9. Nicolas A, Rossignol J-L. Intermediates in homologous recombination revealed by marke effects in Ascobolus. Genome 1989; 31: 528-35. 10. Nicolas A, Treco D, Schultes NP, Szostak JW. An initiation site for meiotic gene conversion in the yeast Saccharomyces cerevisiae. Nature $1989 ; 338: 35-9$

11. Sun H, Treco D, Schultes NP, Szostak JW Double-strand breaks at an initiation site for meiotic gene conversion. Nature $1989 ; 338: 87-90$.

12. Fabre F, Roman H. Genetic evidence for the inducibility of recombination competence in yeast. Proc Natl Acad Sci USA 1977 ; 74 : 1667-71.

13. Hawley RS. Exchange and chromosomal segregation in eucaryotes. In : Smith GR Kucherlapati R, ed. Genetic Recombination. Washington DC : American Society for Microbiology, 1988 : 497-527.

14. Alani E, Padmore R, Kleckner N. Analysis of wild-type and rad50 mutants of yeast suggests an intimate relationship between meiotic chromosome synapsis and recombination. Cell $1990 ; 61$ (in press).

15. Reynaud CA, Anquez V, Grimal H, Weill $\mathrm{J}$-C. A hyperconversion mechanism generates the chicken light chain preimmune repertoire. Cell 1987 ; 48 : 379-88.
Recombinaison génétique : processus conduisant à un changement dans l'association physique entre deux segments d'ADN.

Recombinaison homologue (ou recombinaison générale) : processus de recombinaison exigeant un minimum d'homologie de séquence entre les deux partenaires et pouvant s'effectuer en n'importe quel point $d u$ génome.

Recombinaison ectopique : recombinaison entre deux segments d'ADN situés en des emplacements différents du génome, qui peut avoir lieu quand le génome comporte des séquences répétées dispersées.

Recombinaison homotopique : recombinaison entre deux séquences situées au même locus (au même emplacement dans le génome).

Conversion : transfert unidirectionnel d'information d'un duplex d'ADN parental vers l'autre.

Crossing-over: échange réciproque entre les segments d'ADN situés de part et d'autre de la région d'interaction.

$A D N$ hybride: segment de molécule d'ADN constitué de deux brins complémentaires issus chacun d'un duplex d'ADN parental différent.

$A D N$ hétéroduplex : $A D N$ hybride issu d'ADN parentaux différant par une ou plusieurs mutations et comportant de ce fait au moins un mésappariement de bases (par exemple, $G-T$ ou $A-C)$.

16. Maizels N. Might gene conversion be the mechanism of somatic hypermutation of mammalian immunoglobin genes? Trends in Genetics $1989 ; 5: 4-8$

17. Szostak JW, Wu R. Unequal crossing over in the ribosomal DNA of Saccharomyces cerevisiae. Nature $1980 ; 284: 426-30$.

18. Lichten M, Borts RH, Haber JE. Meiotic gene conversion and crossing-over between dispersed homologous sequences occurs frequently in Saccharomyces cerevisiae. Genetics 1987 ; 115 : 233-46. 19. Rayssiguier C, Thaler D, Radman M. The barrier to recombination between Escherichia coli and Salmonella typhimurium is disrupted in mismatchrepair mutants. Nature 1989 ; 342 : 396-401. 20. Cambareri EB, Jensen BC, Schabtach E, Selker EU. Repeat-induced G-C to A-T mutations in Neurospora. Science 1989 ; 244: 1571-5.

21. Goyon C, Faugeron G. Targeted transformation of Ascobolus immersus and de novo methylation of the resulting duplicated DNA sequences. $\mathrm{Mol}$ Cell Biol 1989; 9 : 2818-27.

22. Faugeron G, Rhounim L, Rossignol J-L. How does the cell count the number of ectopic copies of a gene in the premeiotic inactivation process acting in Ascobolus imersus? Genetics 1990 ; 124: $585-91$.

23. Radman M, Wagner R. The High Fidelity of DNA Duplication. Scientific American 1988: 259: 24-30. 